\title{
Foudroyanter Verlauf eines Plattenepithelkarzinoms auf chronischer Ulzeration
}

\section{Foudroyant Course of a Squamous-Cell Carcinoma on Chronic Ulceration}

Autoren

Institut

\section{E. Rohde, D. Trebing, Y. Kröning, M. Brunner, C. C. Zouboulis}

Klinik für Dermatologie, Venerologie und Allergologie/Immunologisches Zentrum (Chefarzt: Prof. Dr. med. Ch. C. Zouboulis), Städtisches Klinikum Dessau, Akademisches Lehrkrankenhaus der Martin-Luther-Universität Halle-Wittenberg

\section{Bibliografie}

DOI $10.1055 / \mathrm{s}-2008-1077703$

Akt Dermatol 2008; 34:

487-489 @ Georg Thieme

Verlag KG Stuttgart · New York ISSN 0340-2541

\section{Korrespondenzadresse}

\section{Dr. Ewa Rohde}

Klinik für Dermatologie, Venerologie und Allergologie/ Immunologisches Zentrum des Städtischen Klinikums Dessau Auenweg 38

06847 Dessau-Roßlau ewa.rohde@klinikumdessau.de

\section{Zusammenfassung \\ $\nabla$}

Bei dem 67-jährigen Patienten bestand seit 3 Jahren eine chronische, nicht heilende Ulzeration des Zehenzwischenraumes D4/D5 links. Mehrere Probebiopsien aus dem Ulkus ergaben keinen Hinweis auf Malignität. Vor vier Wochen Entwicklung eines schnell wachsenden Knotens auf der Ulzeration. Es erfolgte die Resektion des 4.

\section{Einleitung \\ $\nabla$}

Mit einer Inzidenz von 20 - 30 Neuerkrankungen pro 100000 Einwohner in Mitteleuropa gehören die Plattenepithelkarzinome nach den Basalzellkarzinomen zu den zweithäufigsten Tumoren der Haut. Bei der Entstehung der Plattenepithelkarzinome spielt die kumulative UV-Exposition bei genetischer Disposition (lichtempfindliche Haut vom Typ I und II nach Fitzpatrick) die wichtigste Rolle. Somit entwickeln sich die Tumore vor allem in den sonnenexponierten Arealen, 90\% entstehen im Gesicht. Zu den weiteren Risikofaktoren gehören chemische Karzinogene (Arsen und Teer) sowie Röntgenstrahlen. Seltener entstehen Plattenepithelkarzinome im Bereich der chronischen Wunden und Entzündungen.

Die Plattenepithelkarzinome entwickeln sich erfahrungsgemäß langsam, wachsen typischerweise lokal destruierend und metastasieren selten (5\% der Patienten). Die 5-Jahres-Überlebensrate beim Auftreten der Metastasierung beträgt $25-50 \%$.

\footnotetext{
* Nach einem Vortrag gehalten anlässlich des Symposions „Moderne Aspekte der Dermatoonkologie“ organisiert vom Tumorzentrum und der Klinik für Dermatologie, Venerologie und Allergologie - Immunologisches Zentrum, Städtisches Klinikum Dessau, März 2007.
}

und 5. Strahles des linken Fußes, histologisch ergab sich ein sarkomatoides Plattenepithelkarzinom (G3), pT1NoMo. Regelmäßige Nachsorgeuntersuchungen waren unauffällig.

Das Entstehen von Plattenepithelkarzinomen mit einem untypischen klinischen Verlauf in chronischen Wunden sollte differenzialdiagnostisch berücksichtigt und histologisch abgeklärt werden.

\section{Kasuistik \\ $\nabla$}

\section{Anamnese}

Bei dem 67-jährigen Patienten bestand seit drei Jahren eine chronische, therapieresistente Ulzeration des Zehenzwischenraumes D4/D5 links. Nebenbefundlich bestanden eine Polyneuropathie und ein arterieller Hypertonus. Nach mikrobiologischer Diagnostik und histologischem Ausschluss eines Malignoms wurde die Diagnose eines chronischen superinfizierten Ulkus gestellt. Trotz konsequenter Therapie mit desinfizierenden, antibiotischen und antimykotischen Maßnamen sowie zweimaliger Spalthautdeckung zeigte das Ulkus über Jahre keine Heilungstendenz. Engmaschige Kontrollen des Patienten in unserer Ambulanz mit mehrmalig wiederholter umfangreicher Diagnostik (mikrobiologische und histologische Untersuchungen, Röntgenund MRT-Untersuchung des linken Fußes) ergaben bisher keinen Anhalt für das Vorliegen eines malignen Prozesses. Seit der letzten Vorstellung vor vier Wochen kam es im Bereich des linken Vorfußes zum Entstehen eines schnell wachsenden, exophytischen, erythematösen Knotens mit zentraler Ulzeration.

\section{Aufnahmebefund}

Bei der Aufnahme sehen wir einen 67-jährigen Patienten im guten Allgemeinzustand. Im Bereich des Zehenzwischenraumes D4/D5 links be- 


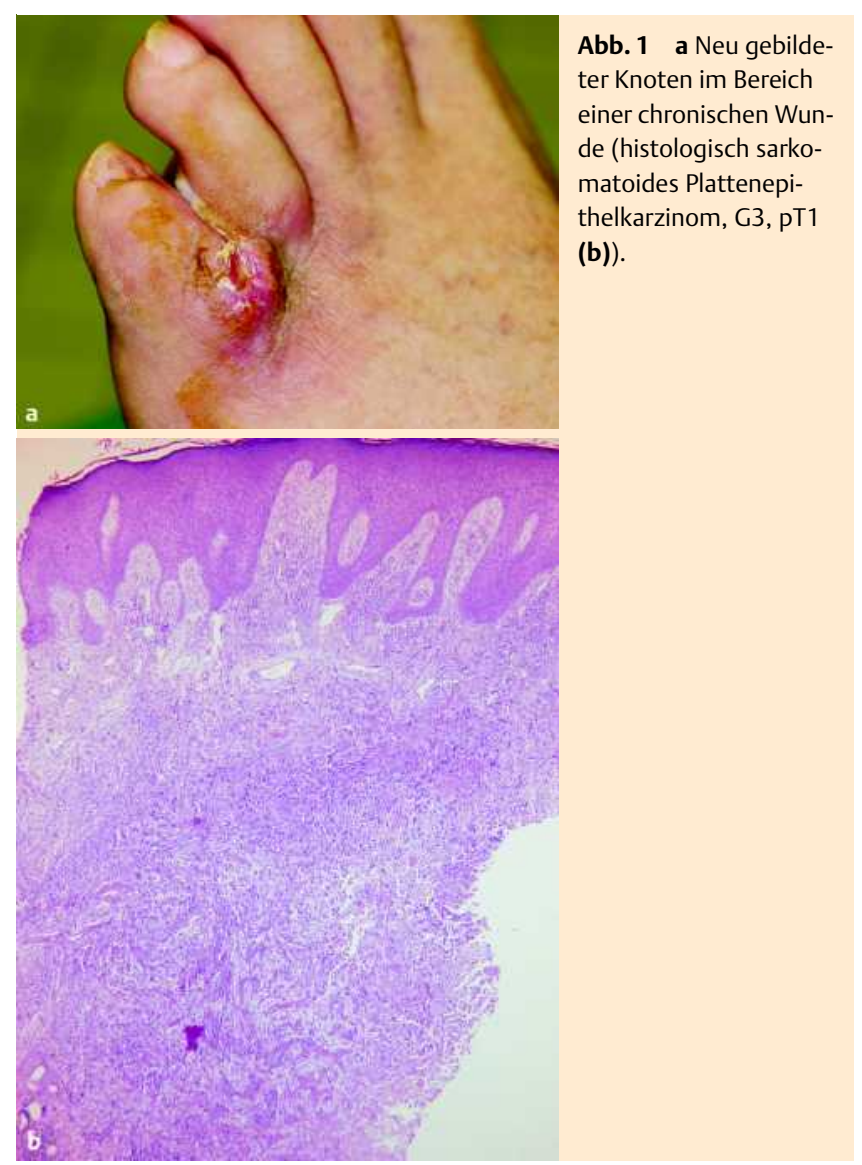

findet sich die bereits bekannte Ulzeration, die sich auf die beiden angrenzenden Zehen D4 und D5 lateral erstreckt. Das Ulkus ist mit Fibrin belegt und deutlich fötide. Es zeigt sich ein livides Erythem der Ulkusumgebung. Zusätzlich fällt ein im Vergleich zur Voruntersuchung vor 4 Wochen neu aufgetretener Knoten im Bereich des ZZR zum Fußrücken hin ( $\bullet$ Abb. 1) auf. Dieser ist hautfarben bis erythematös, derb und nicht verschieblich. Eine Vergrößerung der inguinalen Lymphknoten lässt sich klinisch nicht feststellen.

\section{Befunde diagnostischer Untersuchungen}

Mikrobiologische Untersuchungen des Abstriches aus dem Ulkusgrund ergaben die Besiedlung mit Proteus mirabilis. Der Abstrich aus Hefe- bzw. Schimmelpilzen war negativ. Das Routinelabor inkl. Blutbild, Diff.-BB, Gerinnungsparameter, Elektrolyte, GOT, GPT, $\gamma$ GT, Creatinin, Harnstoff, Blutzucker, CRP) war unauffällig. Histologische und immunhistologische Untersuchungen der Probeentnahme aus dem Knoten ergaben die Diagnose eines sarkomatoiden Plattenepithelkarzinoms (G3) im Bereich des linken Vorfußes.

\section{Ergänzende apparative Untersuchungen}

Die Röntgenuntersuchung des linken Vorfußes in zwei Ebenen zeigte keinen Nachweis von Osteolysen. Die aktuelle MRT-Untersuchung des linken Fußes zeigte im Bereich der 4. und 5. Zehe li. Weichteilschwellung mit diffusen KM-Anreicherungen und Verdickung der Kutis und Subkutis ohne den Hinweis auf artikuläre oder ossäre Beteiligung. Die Staging-Untersuchungen inkl. Röntgenuntersuchung des Thorax sowie Ultraschalluntersuchung des Abdomens und der Lymphknoten waren bis auf die LK-Sonografie, die die beidseitige inguinale Lymphknoten- vergrößerung zeigte, unauffällig. Die histologische Untersuchung des Lymphknotens ergab eine unspezifische Entzündung.

\section{Therapie und Verlauf}

Wir behandelten die bakterielle Superinfektion lokal mit Braunovidon-Lösung und sterilen Verbänden unter Beinhochlagerung und Spreizung des ZZR D4/D5. Systemisch wurde entsprechend dem Antibiogramm mit Rocephin $2 \mathrm{~g}$ i. v. pro Tag über 10 Tage therapiert. Die Thromboseprophylaxe erfolgte mit Clexane $0,2 \mathrm{ml}$ s.c. täglich. Die Therapie der Polyneuropathie wurde nach Rücksprache mit Neurologen unseres Hauses mit Gabapentin p. o. neu eingeleitet. Gegen die Schmerzen erhielt der Patient Novalgin-Tropfen $3 \times$ täglich. Insgesamt hatte sich unter dieser Therapie der entzündliche Zustand deutlich gebessert, sodass der Patient bald einer operativen Therapie unterzogen werden konnte. Diese bestand in der Amputation des 4. u. 5. Strahles des linken Fußes.

Regelmäßige Nachsorgeuntersuchungen waren bisher unauffällig.

\section{Diskussion \\ $\nabla$}

Plattenepithelkarzinome nehmen ihren Ursprung von den epidermalen Stammzellen der intrafollikulären Epidermis. Die bisherigen Untersuchungen machen deutlich, dass Plattenepithelkarzinome molekulargenetische Veränderungen aufweisen, die an verschiedenen Stellen insbesondere die Regulation des Zellzyklus und der Apoptose betreffen und somit die Tumorzellen befähigen, sich der normalen Wachstumskontrolle zu entziehen. Der wichtigste ätiologische Faktor ist die chronische UV-Exposition insbesondere bei UV-empfindlichen Individuen. Das individuelle Tumorrisiko wird außer durch den Hauttyp und die UVExposition auch durch DNA-Sequenzpolymorphismen erheblich mitbestimmt [1].

Zusätzlich zu den genetischen Veränderungen sind wahrscheinlich auch Beeinträchtigungen der T-Zell-vermittelten Immunüberwachung an Ätiologie und Pathogenese beteiligt. Bei Immunsuppression ist die Inzidenz stark erhöht und die Krankheitsverläufe sind ungünstiger. Bei immunsupprimierten Patienten wird die maligne Transformation durch eine erhöhte Infektionsrate mit kanzerogenen humanen Papillomvirustypen getriggert [2].

Neben der malignen Transformation durch UV-Strahlung können bei der Entstehung der Plattenepithelkarzinomen auch andere Faktoren eine Rolle spielen. Dazu gehören Arsenexposition und Röntgenstrahlung sowie chronische Wunden und Entzündungen wie Ulcera crurum, Narben, Verbrennungen, lichenoide Erkrankungen und bullöse Dermatosen [3].

Die Möglichkeit des Auftretens von Plattenepithelkarzinomen auf chronischen Wunden ist mit einer Prävalenz von 1:5000 Wunden eher selten. Das klinische Bild - von kaum sichtbaren Veränderungen bis zu ausgedehnten Tumoren - und der Zeitpunkt des Auftretens können dabei sehr unterschiedlich sein [4]. Die Prognose des Plattenepithelkarzinoms ist abhängig von einer Reihe von Faktoren wie Lokalisation und Größe des Tumors, Eindringtiefe und Differenzierungsgrad des Tumors.

Bei dem vorgestellten Fall handelt es sich zum einen um eine seltene Lokalisation und zum anderen um eine ungewöhnlich schnelle Entwicklung eines Plattenepithelkarzinoms im Bereich einer chronischen Wunde. Die in histologischen Untersuchungen nachgewiesene Entdifferenzierung dieses sarkomatoiden 
Plattenepithelkarzinoms könnte den foudroyanten Verlauf des Tumors erklären.

Da chronische Wunden diverser Genese sehr häufig anzutreffen sind, muss bei länger bestehenden Wunden differenzialdiagnostisch immer an die Möglichkeit der Entwicklung eines Plattenepithelkarzinoms gedacht werden. Engmaschige Kontrollen mit mehrmaligen Probebiopsien erlauben eine rechtzeitige Erkennung des Tumors und tragen wesentlich zu einer besseren Prognose der Erkrankung bei.

\section{Abstract}

\section{Foudroyant Course of a Squamous-Cell Carcinoma on Chronic Ulceration}

\section{$\nabla$}

The 67-years-old patient has been suffering from a chronic ulceration of the interdigital space D4/D5 of the left foot for three years. Multiple exploratory excisions from the ulcer made at different times had always excluded malignity. Four weeks ago a quickly growing node had developed in the region of the left forefoot. The treatment was done by surgical excision. The histological finding showed squamous-cell carcinoma (G3), pT1NoMo. Staging examinations were all free from metastases. The genesis of squamous-cell carcinoma of atypical clinical progress in chronic wounds should be always considered. If necessary, repeated biopsies should be done to exclude malignancy.

\section{Literatur}

1 Reifenberger J, Schön MP. Epitheliale Tumoren der Haut: Molekulare Grundlagen und pathogenese-orientierte Therapie. Der Hautarzt 2003; 54: $1164-1170$

2 Harwood CA, Surentheran T, McGregor JM. Human papillomavirus and non melanoma skin cancer in immunsuppressed and immunocompetent individuals. J Med Virol 2000; 61: 289-297

3 Motley R, Kersey P, Lawrence C. Multiprofessional guidelines for the management of the patient with primary cutaneous squamous cell carcinoma. Br J Dermatol 2002; 141: 18 - 25

4 Dalgleish AG, O'Byrne $K$. Inflammation and cancer: the role of the immune response and angiogenesis. Cancer Treat Res 2006; 130: 1- 38 